\title{
Teaching the Internet of Things: The first three years
}

\author{
$1^{\text {st }}$ Ryan Grammenos \\ Department of Electronic and Electrical Engineering \\ University College London \\ London, UK \\ r.grammenos@ucl.ac.uk
}

\author{
$2^{\text {nd }}$ Clive Poole \\ Department of Electronic and Electrical Engineering \\ University College London \\ London, UK \\ c.poole@ucl.ac.uk
}

\begin{abstract}
This paper describes our experience of designing and delivering a course on the Internet of Things (IoT) to Masters level students. The course runs intensively over one week with $75 \%$ of the content delivered in a semi-flipped style and with a solid focus on practical, hands-on exercises. The emphasis is on systems integration enabling students to build a complete, working IoT system comprising three layers, namely sensors, connectivity and data analytics. While the course is targeted at graduate students in Electronic and Electrical Engineering (EEE) and Computer Science (CS), our student cohort is very diverse with students having completed their first degree in fields such as Physics, Telecommunications and Information Technology. A unique feature of the course lies in strong collaboration with key industrial partners, some being actively involved in the course delivery. The course is proving to be a popular option choice and has received strongly positive student feedback.

Index Terms-Internet of Things, IoT, sensors, connectivity, data analytics, cloud development, teaching, practical, hands-on.
\end{abstract}

\section{INTRODUCTION}

The end of the first decade of the 21 st century marks an important milestone at which the number of devices connected to the Internet surpassed the number of people connected to the Internet [1], thus ushering in the era of the "Internet of Things" (IoT).

IoT is not a technology but rather a framework, a shift in paradigm, defining and enabling the transformation of conventional systems into smart and connected products and services. While the first IoT device actually dates back to the 1980's with Carnegie Mellon's connected coke vending machine [2], multiple advancements in technology since then have lowered the entry barriers and paved the way for easy and rapid development of powerful IoT systems. Examples of such enabling technologies include low cost, yet very powerful edge processors, miniature sensors and actuators, orders of magnitude improvement in power efficiency, pervasive wireless networking and powerful but affordable cloud computing. The wide interdisciplinary nature of the IoT paradigm has also highlighted a growing need for graduate engineers capable of designing, implementing, testing and deploying IoT systems tailored specifically to real world applications.

This paper describes our attempt to address this need by offering a new one week postgraduate module on IoT that forms part of the taught Masters programme in the Department of Electronic and Electrical Engineering (EEE) at University College London (UCL). The taught Masters programme is a 12-month full-time modular course programme where multiple mandatory and optional modules are offered across a range of topic areas. Students are required to take eight of these modules followed by a short research project. Each module is generally taught over a period of one week and is followed shortly by an assessment, either in the form of exams or a written individual assignment. The new IoT module was designed to fit into this overall structure and is offered as an elective to all students on the Masters programme.

\section{Course Philosophy and Methodology}

What distinguishes our programme from other courses in this domain is the inclusion of a data analytics and cloud processing element. Existing courses on teaching IoT discuss the integration of embedded systems with networking while placing an emphasis on typically only one of these two aspects [3] [4] [5] [6]. None of them incorporate data analytics into their curriculum, yet a recent report by Analysys Mason [7] highlights that $60 \%$ of the IoT value chain is found in the data collected by the sensors and devices in an IoT system.

Hence, for our programme, we conceptualised the IoT as comprising three distinct but connected disciplines, each with their own knowledge base and subject matter experts, as illustrated in Fig. 1. Throughout the course a systems engineering approach was adopted, meaning an emphasis on the interconnection of conventional embedded systems with modern data analytics, and taking into account the connectivity provided by pervasive low power wireless networking technologies, such as Bluetooth Low Energy (BLE), LoRa/LoRaWAN and $\mathrm{TSCH} / 6 \mathrm{TiSCH}$.

\section{A. Background}

From the beginning we considered it important that the IoT module should be a "hands-on" module, whereby students would learn the key elements of IoT systems engineering by designing and building an actual working system. We realised early on, however, that due to the wide range of technical disciplines involved in the IoT space, having the students design and build a working IoT system would necessitate a team-based approach, and therefore a method of assessing 


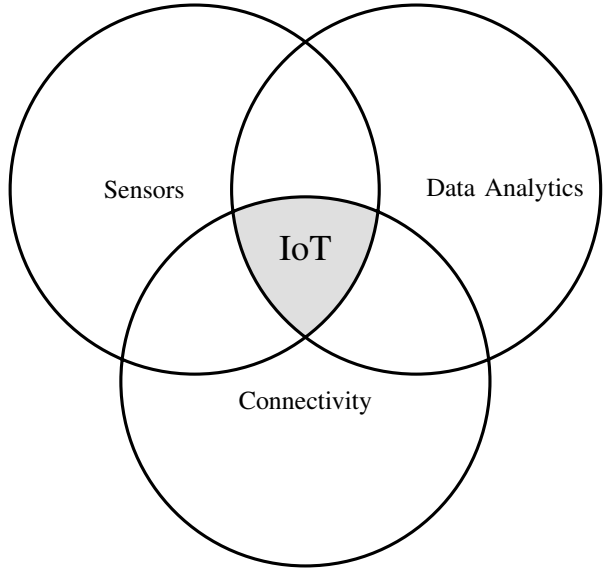

Figure 1. Internet of Things Conceptualisation

team work, as well as individual contributions. It would also not be practical to run such a short duration course without using some "pre-built" sub-system elements to reduce deelopment time, especially in the wireless connectivity area.

We recognised that a course on the Internet of Things needs to accommodate the diverse skills and interest levels of the students. Some will have stronger coding skills whilst others may be more comfortable with hardware or wireless system design. The team-based assessment approach meant that individual students had the opportunity to focus on their strengths, either in the sensor aspect, the connectivity aspect or the data analytics aspect of the project. The team structure also allowed students who have strong leadership, planning and coordination skills to make a positive contribution to their team's overall performance.

The projects were defined with a practical case study in mind, typically inspired by real-world challenges encountered by our industrial partners. Coding workshops were also included to take students gradually from the absolute basics, for example, blinking an LED, all the way through to setting up a basic yet fully functional wireless data link capable of transmitting data to the cloud.

As mentioned, our IoT module is now offered as an elective to all of our students enrolled on our MSc programme. Despite being optional, each year we consistently have close to 40 students signing up for this module, with 51 students signing up in the first year, making it one of the most popular module choices. This cohort size already presents a challenge in itself when compared to the typical class size of 15-25 students reported in similar courses [3] [6]. While the course is targeted to Electronic and Electrical Engineering (EEE) and Computer Science (CS) students, our student cohort in fact has quite a diverse academic background and a wide variation in skill levels, which had to be accounted for during the design and execution of the course.

\section{B. Course structure and objectives}

The course was designed to give postgraduate students who already have a background in electronic engineering, or a related subject, an understanding of what constitutes an IoT application, a solid grounding in the key technologies involved and practical experience of how they can be integrated to form a complete working IoT system. It was intended that the course should have a significant hands-on content, so more than half of the classroom contact time is spent on lab exercises, involving IoT system design and software development. The students are given six weeks to complete a group project. The project is based on one of a selection of scenarios proposed for students to choose from and which requires students to build and demonstrate a simple, yet complete, working IoT system designed to perform specific tasks.

As far as formal learning outcomes are concerned, upon completing the course the students are expected to be able to:

1) Explain the definition and usage of the term "The Internet of Things" in different contexts.

2) Appreciate where the IoT concept fits within the broader ICT industry and possible future trends.

3) Outline the various network protocols used in IoT.

4) Be familiar with the key wireless technologies used in IoT systems, such as WiFi, Bluetooth LE, ZigBee (802.15.4) and LoRa.

5) Understand the role of big data, cloud computing and data analytics in a typical IoT system and be able to implement a basic cloud-based data collection and analytics engine.

6) Design, build and test a simple IoT system comprising sensors and actuators, wireless network connectivity, data analytics, as well as necessary control and data processing software.

Table I sets out the agenda of contact sessions that make up the course. Alongside the technical sessions, two short lectures on business considerations and legal challenges have also been included to provide a broader commercial perspective to the application of IoT technology.

\section{TOOls, Platforms AND Resources}

When selecting the design tools to be used in teaching IoT, we took into account several aspects including power consumption, the number and type of on-board sensors and peripherals, as well as the level and quality of support and documentation available. From an educational perspective, the ease of use of the integrated development environment (IDE) available for the software tools was also an extremely important consideration.

Based on the above requirements, we chose the lowcost (\$30) Texas Instruments (TI) SimpleLink Wi-Fi CC3200 LaunchPad as the edge device, which integrates the highperformance ARM Cortex-M4 microcontroller unit (MCU). This LaunchPad also has on-chip Wi-Fi, features multiple LEDs, sensors and push-buttons, and can operate from two AA batteries. Another advantage of this board is that it can be programmed using two different IDEs; either the "Arduinostyle" Energia IDE for basic users, or Code Composer Studio (CCS) for advanced users. 
Table I

COURSE AGENDA

\begin{tabular}{|c|c|}
\hline $\begin{array}{l}\text { Introduction to the In- } \\
\text { ternet of Things }\end{array}$ & $\begin{array}{l}\text { - What is the IoT and why is it } \\
\text { important? } \\
\text { - Elements of an IoT ecosystem. } \\
\text { - Technology and business drivers. } \\
\text { - IoT applications, trends and im- } \\
\text { plications. }\end{array}$ \\
\hline  & $\begin{array}{l}\text { - Sensing components and devices. } \\
\text { - Sensor modules, nodes and sys- } \\
\text { tems. }\end{array}$ \\
\hline $\begin{array}{l}\text { Connectivity and net- } \\
\text { works }\end{array}$ & $\begin{array}{l}\text { - Wireless technologies for the IoT. } \\
\text { - Edge connectivity and protocols. } \\
\text { - Wireless sensor networks. }\end{array}$ \\
\hline $\begin{array}{l}\text { Analytics and applica- } \\
\text { tions }\end{array}$ & $\begin{array}{l}\text { - Signal processing, real-time and } \\
\text { local analytics. } \\
\text { - Databases, cloud analytics and } \\
\text { applications. }\end{array}$ \\
\hline Industry perspective & $\begin{array}{l}\text { - Business considerations. } \\
\text { - Legal challenges. }\end{array}$ \\
\hline IoT lab exercises & $\begin{array}{l}\text { - Local processing on the sensor } \\
\text { nodes. } \\
\text { - Connecting devices at the edge } \\
\text { and to the cloud. } \\
\text { - Setting up wireless mesh net- } \\
\text { works. } \\
\text { - Processing data offline and in the } \\
\text { cloud. }\end{array}$ \\
\hline
\end{tabular}

Examples of sensors used in conjunction with the CC3200 LaunchPad include temperature, humidity, accelerometer, passive infrared (PIR) and sound sensors. These sensors are connected to the CC3200 MCU via a variety of interfaces including general purpose input output (GPIO), analogue to digital (ADC), as well as digital data, for example interintegrated circuit (I2C) and serial peripheral interface (SPI).

To introduce students to connectivity and networking, we chose the Analog Devices SmartMesh IP kit [8]. Whilst expensive ( $\$ 750$ for the starter kit), this off-the-shelf solution provides a reliable, robust and scalable solution for teaching students low power wireless mesh networking. Furthermore, this kit is supported by a comprehensive and very wellmaintained wiki called Dust Academy [9].

For measurement purposes, students have the option of choosing one of the following portable USB laboratories; the National Instruments (NI) myDAQ, the Analog Discovery 2, or the Pico Technology DrDAQ. All three options, which range between $\$ 130$ and $\$ 190$, provide basic tools, such as power supplies, voltmeters, oscilloscopes and waveform generators.

Finally, IBM Cloud was our cloud platform of choice, thanks to the very strong collaboration and one-to-one support offered by our partners at IBM. IBM Cloud offers CloudantDB and DashDB, as popular NoSQL and SQL data storage methods, respectively. NodeRED is a superb tool for "wiring-up" things connected to the Internet. The Watson IoT platform allows devices to be registered on IBM Cloud, thus adding authentication and encryption. Watson Studio offers three options for carrying out data analytics in the cloud; either $\mathrm{R}$ or Python for advanced users, SPSS Modeler for beginner to intermediate users, or Neural Network Modeler for automated deep learning. Through IBM's Academic Initiative program, all the above are offered to academic staff and students free of charge. Moreover, a number of datasets are made available to us freely both through our IBM colleagues and the IBM online community.

Table II summarises the tools and partners used according to the IoT stack layer.

Table II

LAYERS, TOOLS AND PARTNERS

\begin{tabular}{lll}
\hline IoT Layer & Tools & $\begin{array}{l}\text { Technology } \\
\text { partner }\end{array}$ \\
\hline $\begin{array}{l}\text { Sensing and edge } \\
\text { computing }\end{array}$ & $\begin{array}{l}\text { CC3200 } \\
\text { LaunchPad }\end{array}$ & Texas Instuments \\
\hline $\begin{array}{l}\text { Connectivity and } \\
\text { networking }\end{array}$ & SmartMesh IP & $\begin{array}{l}\text { Analog Devices, } \\
\text { Inria (France) }\end{array}$ \\
\hline $\begin{array}{l}\text { Data analytics and } \\
\text { presentation }\end{array}$ & $\begin{array}{l}\text { IBM Cloud, } \\
\text { MATLAB }\end{array}$ & IBM, Mathworks \\
\hline
\end{tabular}

\section{IMPLEMENTATION}

The hands-on workshops are organised into three categories where each category addresses a specific layer of the IoT stack. The first workshop involves programming the CC3200 LaunchPad using a simplified version of the $\mathrm{C}$ programming language. Students are shown how to connect sensors and actuators to the development board, and subsequently send sensor data to the cloud. This workshop helps students appreciate the constraints imposed on the overall system design as a result of the limited physical resources (for example, memory, processing speed) typically available on an IoT device, such as the CC 3200 .

The second workshop uses the SmartMesh IP connectivity solution to setup low power wireless mesh networks. This workshop introduces students to wireless sensor networks (WSNs), demonstrating the heterogeneous landscape of WSNs and helping them evaluate network performance. While the students are not required to design any wireless protocols, as such, they need to make use of the Dust Academy wiki page, which introduces them to important design parameters and trade-offs at the medium access control (MAC) layer, for example, latency, throughout, reliability, stability, scalability and power consumption.

The third workshop focuses on data analytics, both offline and online. Offline analytics are executed in MATLAB while online analytics are carried out in IBM's Watson Studio platform. Whilst MATLAB is a proprietary software package (for which UCL holds a campus-wide licence), it was chosen for offline data analytics for two key reasons; firstly, the academic 
staff teaching on the course share decades of experience in using MATLAB for their teaching and research, thereby allowing them to respond to student needs more effectively and efficiently compared to trending software tools like $\mathrm{R}$ or Python; secondly, MATLAB has an unrivalled library of fullyfunctional and thoroughly tested functions supported by comprehensive documentation. For online analytics, IBM Cloud makes use of open-source tools to avoid licencing barriers typically encountered with proprietary software. Hence, IBM Cloud allows Python notebooks and $\mathrm{R}$ scripts to be compiled and executed within their online platform. Developers familiar with all three programming languages (MATLAB, Python, R) will be aware that these languages share similar syntax and hence, it is fairly straightforward for students to implement the same pseudocode in either of the three languages.

By the end of the three workshops, the students will have acquired all the "know-how" needed to design and build an end-to-end IoT system including embedded processing at the edge followed by data analytics and application development in the cloud. Many successful small IoT projects have been thus created, and short videos of some of our previous students' project outcomes can be viewed on UCL's MediaCentral platform by entering the search string "MSc IoT" [10].

As one example, Figures 2-4 showcase the implementation of the three IoT layers for a smart parking project developed by one of the student teams. Specifically, Fig. 2 illustrates the edge layer comprising a CC3200 LaunchPad and four ultrasonic promixity sensors with block diagrams emulating a real-world scenario. Fig. 3 demonstrates that the sensor data has been transmitted to the cloud successfully with NodeRED "linking" the edge to the cloud. A simple cloud data analytics model was then developed in SPSS and the predicted outcomes (available parking slots) are depicted in a mobile app (also created by the students), as shown in Fig. 4.

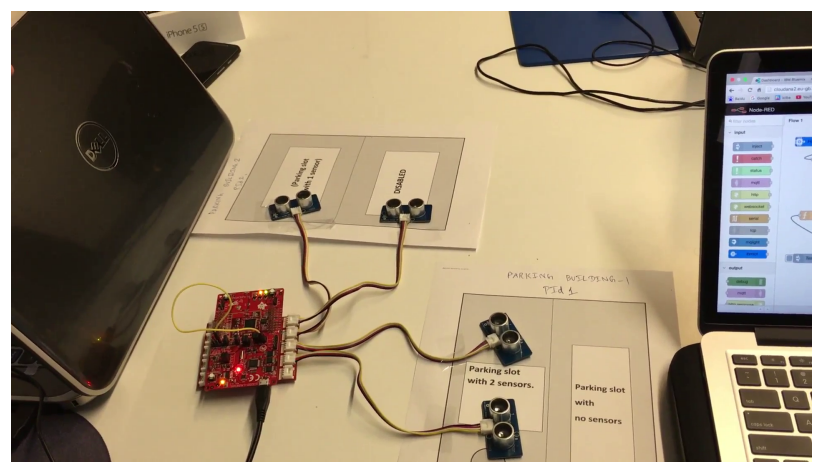

Figure 2. Edge implementation for smart parking project

\section{Evaluation}

Feedback on the course is canvassed by means of a form given to the students to complete at the end of the module. The feedback form records students views as to whether the module had achieved overall objectives, as well as the performance of individual instructors. Students entered their scores using a Likert scoring system of 1 to 5 .



Figure 3. NodeRED wiring linking sensor data to the cloud



Figure 4. Mobile app showing forecasting results

Fig. 5 illustrates the students' impressions of the course quality, in terms of whether the course met overall objectives, the quality of the course materials and the knowledge level of the instructors, over the first three years, with a score of 5 representing highest quality.

Fig. 5 shows that the instructors' knowledge (beige bar) ranks high consistently with an average of 4.4 / 5 over the three years. With regard to achieving the course objectives (blue bar), we observe a sudden drop in 2017. This was a result of making the course more challenging in 2017 compared to 2016. Specifically, the instructors felt that the students had not acquired a comprehensive appreciation of all IoT layers in 2016, particularly around data analytics. Therefore, more material and additional project deliverables (including a viva) were introduced in 2017 which overwhelmed the students. To address this issue, we "semi-flipped" the classroom in 2018, moving more of the theoretical material offline and introducing more practical and hands-on exercises during the contact hours with the students. This action restored the achievement of the course objectives back up to 4 / 5 on the Likert scale. Finally, regarding the course material (red bar), students evaluated this at an average of 3.9 / 5 over the three years. As mentioned above, the course material became more challenging and the structure of the course more intensive in 2017 and 2018 compared to 2016, which explains the drop from 4.1 to 3.8 in 2017 and 2018.

Fig. 6 shows the students' impressions of the difficulty of the course material and the pace of teaching over the first three 
years. They scored these two aspects on a 3-point Likert scale, with 1 being difficult and fast-paced, 2 being just right, and 3 being easy and slow-paced. Fig. 6 corroborates the trends shown in Fig. 5. That is, students found both the difficulty of the material (blue bar) and the pace of teaching (red bar) somewhat on the easier side (score higher than 2) in 2016. We then observe a drop in 2017 during which students found the course material too difficult and the pace of teaching too fast, as a result of making the course more challenging. Finally, in 2018, the scores are restored to the expected outcomes (an average score of $2 / 3$ ), thanks to the positive changes that were introduced.

Fig. 6 in particular demonstrates that by the third year, we achieved in setting the difficulty of the course and the pace of teaching at "just the right" level for our student cohort. To conclude, the most useful aspects of the course may be summarised in some of the quotes provided by students over the three years, which were taken verbatim from the student feedback forms:

- Excellent lectures from industry experts and hands-on knowledge

- Excellent course - well planned labs

- IBM cloud and TI CC3200 - new to this platform. Great practical view of IoT and developing practical applications

- We covered the whole framework of IoT

- Workshop and using cases to see the different uses of IoT

- I knew about sensors, cloud analytics from the internet this course achieved the objectives by stitching them all together

- I am impressed. Please do not change the style of IoT teaching. Excellent coverage

- Concepts and accessibility to materials and tools

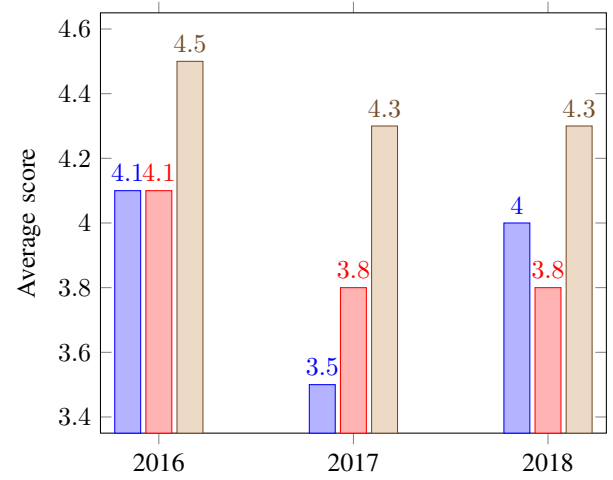



Figure 5. Student feedback - Achievement and delivery

\section{CONCLUSION}

The postgraduate module on IoT described in this paper has been carefully created to address the need for precisely the kind of competent, rounded, systems-oriented engineers needed to design real-world IoT systems to meet the needs of

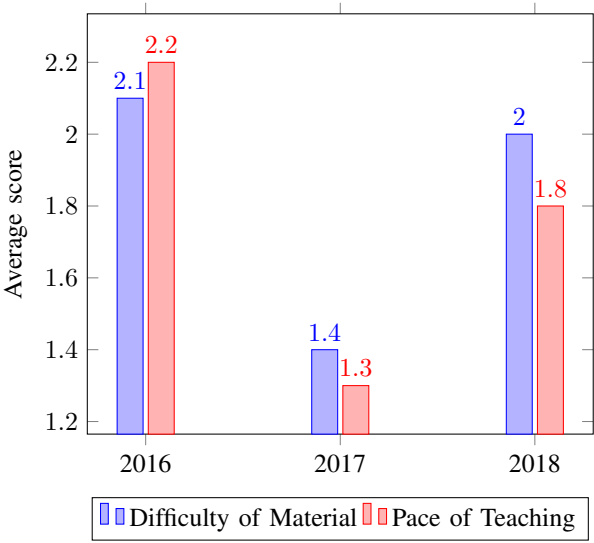

Figure 6. Student feedback - Pace and difficulty

today's industries. The unique structure of the course and a careful selection of tools and technology partners has allowed us to cover the three layers of any IoT sytem (Sensors, Connectivity and Data Analytics) to a sufficient depth to enable students to start designing and building working systems by the end of the one week of contact time. Students also will need to have completed the project over the following 6 weeks to demonstrate mastery of the necessary skills. The module has been consistently one of the most popular option choices for Masters students at UCL, and has received strongly positive feedback from the students.

\section{REFERENCES}

[1] F. Mattern and C. Floerkemeier, "From the internet of computers to the internet of things," Lecture Notes in Computer Science, vol. 6462 LNCS, pp. 242-259, 2010.

[2] J. Teicher, "The little-known story of the first IoT device," Feb 2018. [Online]. Available: https://www.ibm.com/blogs/industries/ little-known-story-first-iot-device/

[3] N. He, R. Bukralia, and H. Huang, "Teaching wireless networking technologies in the internet-of-things using ARM based microcontrollers," in 2017 IEEE Frontiers in Education Conference (FIE). IEEE, Oct 2017, pp. 1-4.

[4] S. G. Koo, "An integrated curriculum for Internet of Things: Experience and evaluation," in Proceedings - Frontiers in Education Conference, FIE, vol. 2014. IEEE, Oct 2015, pp. 1-4.

[5] T. Watteyne, P. Tuset-Peiro, X. Vilajosana, S. Pollin, and B. Krishnamachari, "Teaching Communication Technologies and Standards for the Industrial IoT? Use 6TiSCH!” IEEE Communications Magazine, vol. 55, no. 5, pp. 132-137, May 2017.

[6] C. U. Lei, C. W. Yau, K. S. Lui, P. Yum, V. Tam, A. H.-K. Yuen, and E. Y. Lam, "Teaching Internet of Things: Enhancing learning efficiency via full-semester flipped classroom," in Proceedings of 2017 IEEE International Conference on Teaching, Assessment and Learning for Engineering, TALE 2017, vol. 2018-Janua. IEEE, Dec 2018, pp. 56-60.

[7] T. Rebbeck, "Operators' strategies for IoT are guided by three main motives," Analysys Mason, Jan 2016.

[8] Analog Devices, "DC9021B Evaluation Board." [Online]. Available: https://www.analog.com/en/design-center/ evaluation-hardware-and-software/evaluation-boards-kits/dc9021b. html $\{\backslash \#\}$ eb-overview

[9] T. Watteyne, "Dust Academy." [Online]. Available: https://dustcloud atlassian.net/wiki/spaces/ALLDOC/pages/40468511/Dust+Academy

[10] UCL MediaCentral, "MSc IoT Mini-Project," Dec 2018. [Online]. Available: https://mediacentral.ucl.ac.uk/ 